\title{
Molecular mechanisms of cholangiocarcinoma cell inhibition by medicinal plants
}

\author{
SURANG LEELAWAT $^{1}$ and KAWIN LEELAWAT ${ }^{2}$ \\ ${ }^{1}$ Faculty of Pharmacy, Rangsit University, Amphoe Mueang, Pathum Thani 12000; \\ ${ }^{2}$ Department of Surgery, Rajavithi Hospital, Bangkok 10400, Thailand
}

Received January 20, 2016; Accepted October 18, 2016

DOI: $10.3892 / 01.2016 .5488$

\begin{abstract}
Cholangiocarcinoma (CCA) is one of the most common causes of cancer-associated mortality in Thailand. Certain phytochemicals have been demonstrated to modulate apoptotic signaling pathways, which may be targeted for the prevention and treatment of cancer. Therefore, the aim of the present study was to investigate the effect of specific medicinal plants on the inhibition of CCA cell proliferation, and to identify the molecular mechanisms underlying this. A WST-1 cell proliferation assay was performed using an RMCCA1 cell line, and apoptotic signaling pathways were also investigated using a PathScan Stress and Apoptosis Signaling Antibody Array Kit. The cell proliferation assay indicated that extracts from the Phyllanthus emblica fruit pulp (PEf), Phyllanthus emblica seed (PEs), Terminalia chebula fruit pulp (TCf), Terminalia chebula seed (TCs), Areca catechu seed (ACs), Curcuma longa (CL) and Moringa oleifera seed (MOs) exerted anti-proliferative activity in RMCCA1 cells. In addition, the PathScan assay revealed that certain pro-apoptotic molecules, including caspase-3, poly (ADP-ribose) polymerase, checkpoint kinase 2 and tumor protein 53, exhibited increased activity in RMCCA1 cells treated with the aforementioned selected plant extracts, with the exception of PEf. The mitogen-activated protein kinase (MAPK) pathways (including ERK1/2 and p38 MAPK) expression level was significantly increased in RMCCA1 cells pre-treated with extracts of PEs, TCf, CL and MOs. The activation of protein kinase B (Akt) was significantly demonstrated in RMCCA1 cells pre-treated with extracts of TCf, ACs and MOs. In summary, the present study demonstrated that extracts of PEs, TCf, TCs, ACs, CL and MOs exhibited anti-proliferative effects in CCA cells by inducing pro-apoptotic signals and modulating signal transduction molecules. Further studies
\end{abstract}

Correspondence to: Dr Surang Leelawat, Faculty of Pharmacy, Rangsit University, 52/347 Phahonyothin Road, Amphoe Mueang, Pathum Thani 12000, Thailand

E-mail: surang.1@rsu.ac.th

Key words: anticancer, cholangiocarcinoma, medicinal plants, apoptosis, signal transduction in vivo are required to demonstrate the potential applications of specific plant extracts for the treatment of human cancer.

\section{Introduction}

Cholangiocarcinoma (CCA) is one of the most common causes of cancer-associated mortality in Thailand, and is one of the most aggressive types of cancer due to its resistance to chemotherapy and propensity for local and distant invasion (1). Currently, no effective therapy has been identified for the disease, and alternative therapeutic options are urgently required. Certain dietary phytochemicals have been previously observed to exert chemopreventive and anticancer effects, and have been demonstrated to modulate apoptotic signaling pathways, which may be targeted for the prevention and treatment of CCA (2). To the best of our knowledge, the present study is the first to extensively examine the use of phytochemicals in CCA.

Ten different medicinal plants were selected for the current study and evaluated with regard to their cytotoxicity and cytotoxic mechanisms in a CCA cell line. The selected medicinal plants included Phyllanthus emblica fruit pulp (PEf) and seed (PEs), which contains polyphenols and hydrolysable tannin-derived compounds (3); Phyllanthus extracts have been demonstrated to trigger apoptosis and regulate multiple survival signal pathways in some cancer cell types $(4,5)$. The plants also included Curcuma longa (CL), Terminalia chebula fruit pulp (TCf) and seed (TCs), Moringa oleifera seed (MOs), Momordica charantia, Areca catechu seed (ACs), Brassica oleracea var. rubra and Tinospora crispa (TiC). These extracts have previously demonstrated anti-proliferative effects via modulation of the apoptotic pathways (6-14).

Curcumin, derived from CL, has previously been shown to exhibit apoptotic effects in various cancer cell lines through the activation of caspase- 3 and the downregulation of B-cell lymphoma-2 (BCL-2). Curcumin also activates the expression of tumor protein $\mathrm{p} 53$, peroxisome proliferator-activated receptor (PPAR), and other tumor suppressor genes, and downregulates specific oncogenes, including c-Myc, human epidermal growth factor receptor 2 and epidermal growth factor receptor (6). Chebulagic acid from the TCf has previously been demonstrated to induce G1 arrest, inhibit nuclear factor $\kappa$-light-chain-enhancer of activated B cells $(\mathrm{NF}-\kappa \mathrm{B})$ and induce apoptosis in retinoblastoma cells (7). MOs and 
$M$. oleifera leaf extracts have been observed to induce CCA cell apoptosis by inducing reactive oxygen species accumulation and mitochondrial dysfunction (8). M. oleifera leaf extract inhibits pancreatic cancer cells by targeting the $\mathrm{NF}-\kappa \mathrm{B}$ signaling pathway, increasing the efficacy of chemotherapeutic agents in human pancreatic cancer cells (9). Cucurbitae-type triterpene glycosides from $M$. charantia fruit (MCf) inhibit NF- $\kappa \mathrm{B}$ and activate PPAR in HepG2 cells (10). In addition, $M$. charantia leaf extract induces apoptosis through caspase- and mitochondria-dependent pathways in human cancer cells (11). Arecoline from ACs has been demonstrated to induce $\mathrm{HaCaT}$ cell apoptosis by upregulating the expression and activation of cleaved-BH3 interacting-domain death agonist, cleaved-PARP and cleaved-caspase-3 (12). Sulforaphane from red cabbage [B. oleracea var. capitata L.f. rubra (BO)] has been demonstrated to reduce BCL-2 expression levels (antiapoptotic) and reduce the induction of p53, BCL-2-associated $\mathrm{X}$ protein (proapoptotic) and caspase-3 in HEp-2 cells (13). Methanol extract of the T. crispa stem has been observed to promote apoptosis by upregulating caspase-3 expression levels and insulin sensitization, through the inhibition of the insulin-like growth factor-1 receptor in HepG2 cells (14). Additionally, Solanum torvum (ST) and Allium ascalonicum (AA) have demonstrated cytotoxic effects in multiple cancer cell lines $(15,16)$; however, the mechanisms underlying these effects require further study for elucidation.

The aim of the present study was to investigate the roles of specific medicinal plants that inhibit CCA cell proliferation and identify the molecular mechanisms underlying this.

\section{Materials and methods}

Plant materials and extraction procedures. All plant samples including PEf, PEs, TCf, TCs, ACs, BO, CL, ST, AA, MCf pulp, M. charantia seed (MCs), TiC, M. oleifera fruit (MOf) pulp and MOs were purchased from Rangsit market in Amphoe Mueang, Pathum Thani province, Thailand. The fruits were separated into pulp and seed and all of the samples were dried at $55^{\circ} \mathrm{C}$ and ground into powder. Powder samples (15 g) were macerated with $300 \mathrm{ml} \mathrm{95 \%} \mathrm{ethanol} \mathrm{for} 24 \mathrm{~h}$. Extract solutions were subsequently dried by rotary evaporation and freeze-drying, and stored at $-20^{\circ} \mathrm{C}$ until required. The sample extracts were freshly prepared in dimethyl sulfoxide (DMSO) to the concentration of $50 \mathrm{mg} / \mathrm{ml}$ then diluted with HAM's F12 medium (Gibco; Thermo Fisher Scientific, Inc., Waltham, MA, USA) to the concentration of $1-500 \mu \mathrm{g} / \mathrm{ml}$.

Cell proliferation assay. The human CCA cell line RMCCA1 (16) was grown in HAM's F12 medium supplemented with $10 \%$ fetal bovine serum (Gibco; Thermo Fisher Scientific, Inc.) at $37^{\circ} \mathrm{C}$ in a $5 \% \mathrm{CO}_{2}$ humidified atmosphere. For the proliferation assays, cells were seeded into 96-well culture plates at a density of 10,000 cells per well, followed by the addition of sample extracts in various concentrations $(1-500 \mu \mathrm{g} / \mathrm{ml})$ or control medium (Ham's F12 medium containing $0.01 \%$ DMSO). The cells were subsequently incubated for $48 \mathrm{~h}$ prior to the application of Cell Proliferation Reagent WST-1 (Roche Diagnostics GmbH, Mannheim, Germany) according to the manufacturer's instructions. Cell viability was calculated as a percentage, relative to the control cells. The extracts that exhibited anti-proliferative effects were selected for further assays investigating the effects of stress and apoptosis on the RMCCA1 cell line.

Cellular stress and apoptosis signaling assay. RMCCA1 cells were treated with seven selected extracts (PEf, PEs, TCf, TCs, ACs, CL and MOs) prior to using the PathScan ${ }^{\circledR}$ Stress and Apoptosis Signaling Antibody Array kit with a chemiluminescent readout (cat. 12856; Cell Signaling Technology, Inc., Danvers, MA, USA) according to the manufacturer's instructions. The kit facilitated the simultaneous detection of the phosphorylation of 13 signaling molecules, including extracellular signal-regulated kinase (ERK)-1/2 (Thr202/tyr204), protein kinase B (Akt; Ser473), BCL-2-associated death promoter (Bad; Ser136), heat shock protein 27 (HSP27; Ser82), Smad2 (Ser465/467), p53 (Ser15), p38 mitogen activated protein kinase (MAPK; Thr180/Tyr182), stress-activated protein kinases (SAPK)/Jun amino-terminal kinases (JNK; Thr183/Tyr185), checkpoint kinase (Chk) 1 (Ser345), Chk2 (Thr68), NF- $\kappa \mathrm{B}$ inhibitor $\alpha(\mathrm{I} \kappa \mathrm{B} \alpha$ Ser32/36) eukaryotic translation initiation factor 2 subunit $\alpha$ (elF2; Ser51) and transforming growth factor- $\beta$-activated kinase 1 (TAK1) (Ser412). In addition, the kit also allowed for simultaneous detection of 3 cleaved molecules, including poly (ADP-ribose) polymerase (PARP) (Asp214), caspase-3 (Asp175) and caspase-7 (Asp198), and detection of 3 total proteins, including I $\mathrm{B} \alpha$, survivin and $\alpha$-tubulin. Cell lysates were incubated on the slide followed by a biotinylated detection antibody cocktail. Streptavidin-conjugated HRP and LumiGLO ${ }^{\circledR}$ Reagent were then used to visualize the bound detection antibody by chemiluminescence. Images were acquired by briefly exposing the slide to standard chemiluminescent film. The spot intensity was quantified using ImageJ software (National Institutes of Health, Bethesda, MD, USA). The intensity of each signal was calculated relative to the positive control signal of the array kit.

Statistical analysis. The experiments were performed in triplicate and the data are presented as the mean \pm standard deviation. Data between three or more groups were compared using one-way analysis of variance, followed by Dunnett's post hoc test. $\mathrm{P}<0.05$ was considered to indicate a statistically significant result.

\section{Results}

Effects of plant extracts on cell proliferation. Cell proliferation assays were performed in RMCCA1 cells treated with 14 extract samples at concentrations of 1, 10, 100 and $500 \mu \mathrm{M}$ or control medium. After $48 \mathrm{~h}$ of incubation, the results demonstrated that TCs $(500 \mu \mathrm{g} / \mathrm{ml})$, PEf, PEs, TCf, CL and MOs $(100 \mu \mathrm{g} / \mathrm{ml})$, and ACs $(10 \mu \mathrm{g} / \mathrm{ml})$ significantly inhibited RMCCA1 cell proliferation $(\mathrm{P}=0.002,<0.001,<0.001,<0.001$, $0.002,<0.001$ and 0.008 , respectively). However, BO, ST, AA, MCf, MCs, TiC and MOf had no significant inhibitory effect on RMCCA1 cell proliferation, as compared with control cells (P>0.05; Fig. 1). Therefore, PEf, PEs, TCf, TCs, ACs, CL and MOs were selected to further study the mechanisms underlying CCA cell proliferation inhibition. 


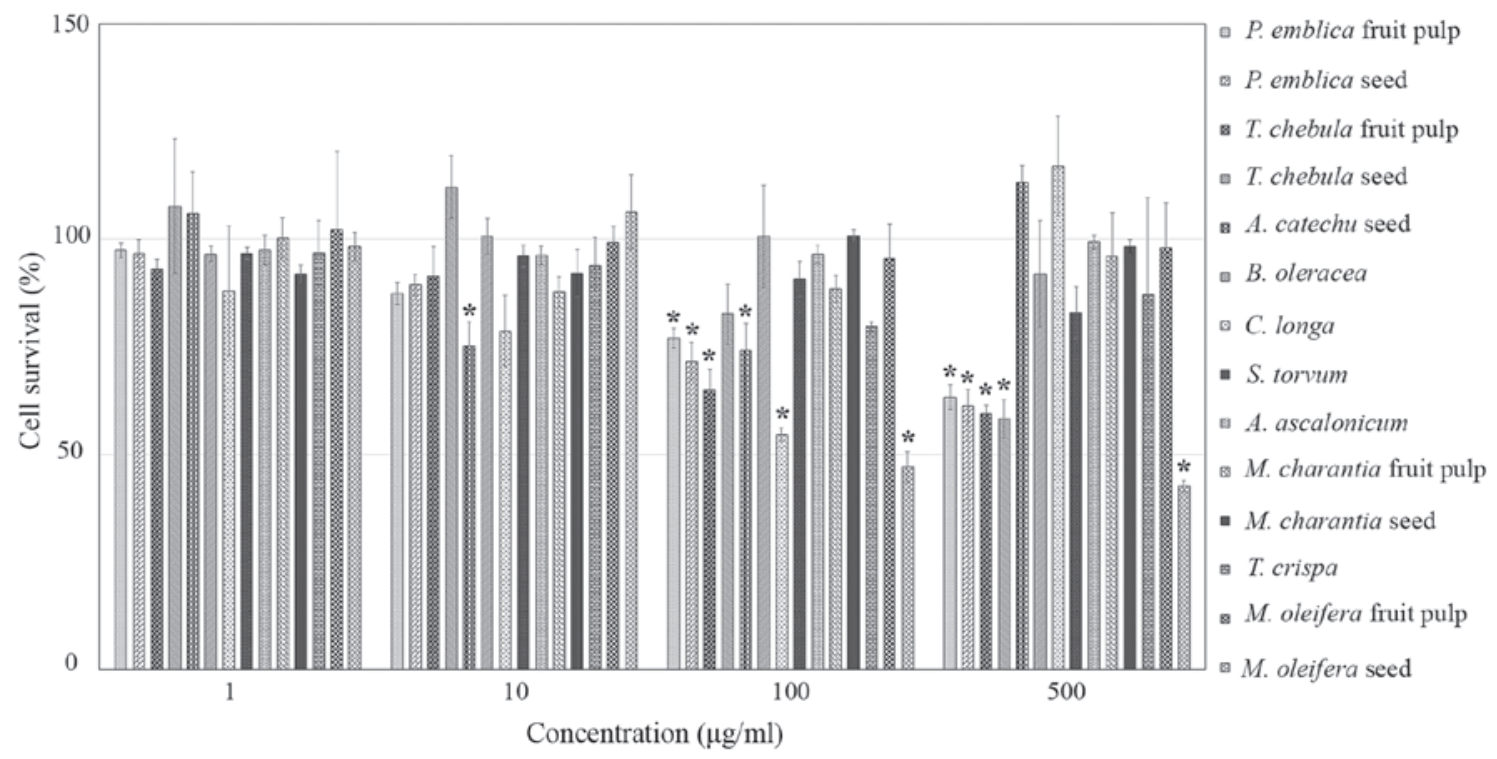

Figure 1. Cell proliferation assay of RMCCA1 cells treated with selected medicinal plants. The cells were treated with extracts of specific medicinal plants at various concentrations for $48 \mathrm{~h}$. Cell proliferation was measured by WST-1 and analyzed by spectrophotometry (absorbance at $450 \mathrm{~nm}$ ). The data are presented as a percentage of cell proliferation, for which the optical density value obtained from control cells, treated with HAM's F12 medium containing $0.01 \%$ Dimethyl sulfoxide, was set at $100 \%$. The results are presented as the mean \pm standard deviation of three independent experiments. ${ }^{*} \mathrm{P}<0.05 \mathrm{vs}$. control.

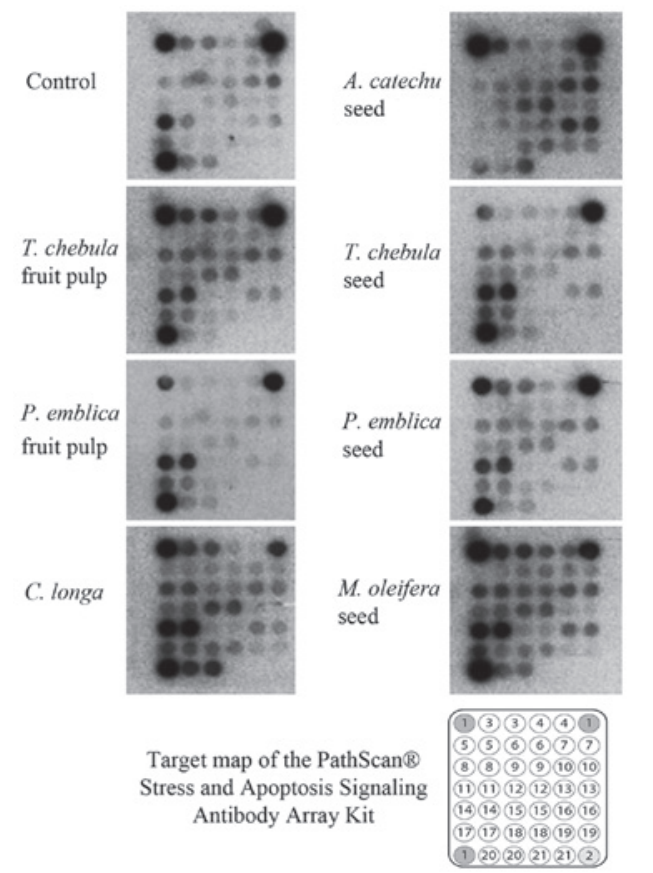

\begin{tabular}{|c|c|c|c|}
\hline \multicolumn{4}{|c|}{ Stress and apoptosis signaling } \\
\hline & Target & Site & Modification \\
\hline 1 & Positive Control & N/A & N/A \\
\hline 2 & Negative Control & $\mathrm{N} / \mathrm{A}$ & N/A \\
\hline 3 & P44/42 MAPK (ERK1/2) & Thr202/Tyr204 & Phosphorylation \\
\hline 4 & Akt & Ser473 & Phosphorylation \\
\hline 5 & Bad & Ser136 & Phosphorylation \\
\hline 6 & HSP27 & Ser82 & Phosphorylation \\
\hline 7 & Smad2 & Ser $465 / 467$ & Phosphorylation \\
\hline 8 & p53 & Ser 15 & Phosphorylation \\
\hline 9 & p38 MAPK & Thr180/Tyr182 & Phosphorylation \\
\hline 10 & $\mathrm{SAPK} / \mathrm{JNK}$ & Thr183/Tyr 185 & Phosphorylation \\
\hline 11 & PARP & Asp214 & Cleavage \\
\hline 12 & Caspase-3 & Asp175 & Cleavage \\
\hline 13 & Caspase-7 & Asp198 & Cleavage \\
\hline 14 & $\mathrm{I} \kappa \mathrm{B} \alpha$ & Total & N/A \\
\hline 15 & Chk1 & Ser345 & Phosphorylation \\
\hline 16 & Chk2 & Thr68 & Phosphorylation \\
\hline 17 & $\mathrm{I} \kappa \mathrm{B} \alpha$ & Ser $32 / 36$ & Phosphorylation \\
\hline 18 & $\operatorname{eIF} 2 \alpha$ & Ser51 & Phosphorylation \\
\hline 19 & TAK1 & Ser 412 & Phosphorylation \\
\hline 20 & Survivin & Total & $\mathrm{N} / \mathrm{A}$ \\
\hline 21 & $\alpha$-Tubulin & Total & N/A \\
\hline
\end{tabular}

Figure 2. The effects of medicinal plants on the phosphorylation or cleavage of certain signaling proteins in RMCCA1 cells. Chemiluminescent array images obtained using the PathScan Stress and Apoptosis Signaling Antibody Array Kit allowed for the simultaneous detection of 19 signaling molecules that involved in the regulation of the stress response and apoptosis. Images were captured following brief exposure of the slide to a standard chemiluminescent film. Numbers on the target map correspond to the numbered targets shown on the right. The expression levels of $\alpha$-tubulin were used to normalize the signals between various samples.

Effects of plant extracts on cellular stress and apoptosis signaling. A PathScan ${ }^{\circledR}$ Stress and Apoptosis Signaling Antibody Array kit with a chemiluminescent readout was used for the simultaneous detection of 19 signaling molecules that are involved in the regulation of the cellular stress response and apoptosis. The expression levels of $\alpha$-tubulin were used to normalize the signals between various samples, and the $\alpha$-tubulin levels were observed to be relatively constant between the samples.
The RMCCA1 cells treated with PEs, TCf, TCs, ACs, CL or MOs exhibited high intensity of the apoptotic signals p53, PARP and caspase-3 whereas PEf did not appear to induce apoptotic signals (Fig. 2). The intensity of each signal was measured by ImageJ software. The expression level was calculated relative to the positive control signal as intensity ratio.

Caspase- 3 and -7 proteases exert pro-apoptotic functions through the cleavage of a number of cellular targets (18). 


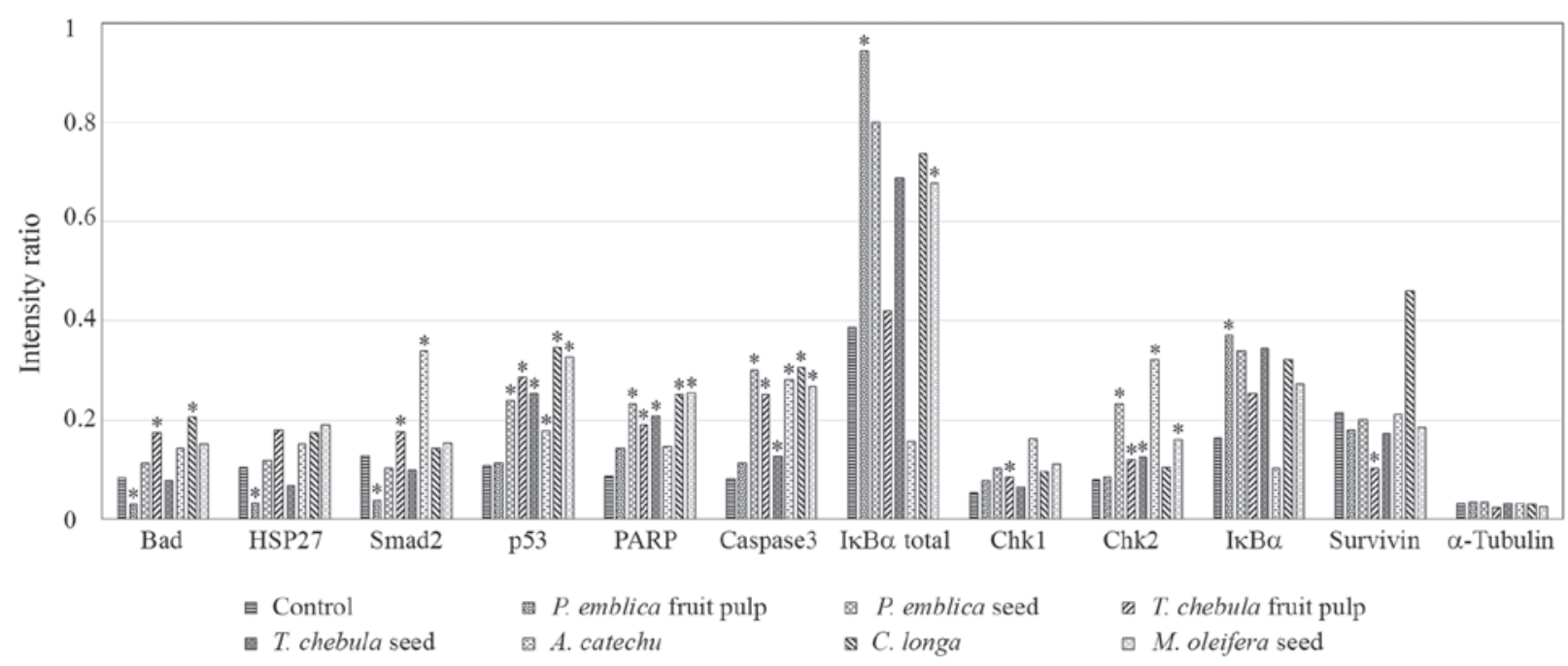

Figure 3. Array image pixel intensity ratio of phosphorylated/cleaved pro-apoptotic signaling molecules in RMCCA1 cells treated with medicinal plants. RMCCA1 cells were treated with seven selected plants that had exhibited apoptotic signals. The phosphorylation levels of Bad at Ser136, HSP27 at Ser82, Smad2 at Ser465/467, p53 at Ser15, IкB $\alpha$ at Ser32/36, Chk1 at Ser345 and Chk2 at Thr68, and the cleavage of PARP at Asp214 and caspase-3 at Asp175, were significantly altered. The expression of survivin decreased only in RMCCA1 cells treated with the extract of TCf. The expression levels of $\alpha$-tubulin were used to normalize the signals between various samples, and these were observed to be consistent. " $\mathrm{P}<0.05$ vs. control. Bad, BCL-2-associated death promoter; HSP27, heat shock protein 27; PARP, poly (ADP-ribose) polymerase; IкB $\alpha, \mathrm{NF}-\kappa \mathrm{B}$ inhibitor $\alpha$; Chk, checkpoint kinase.

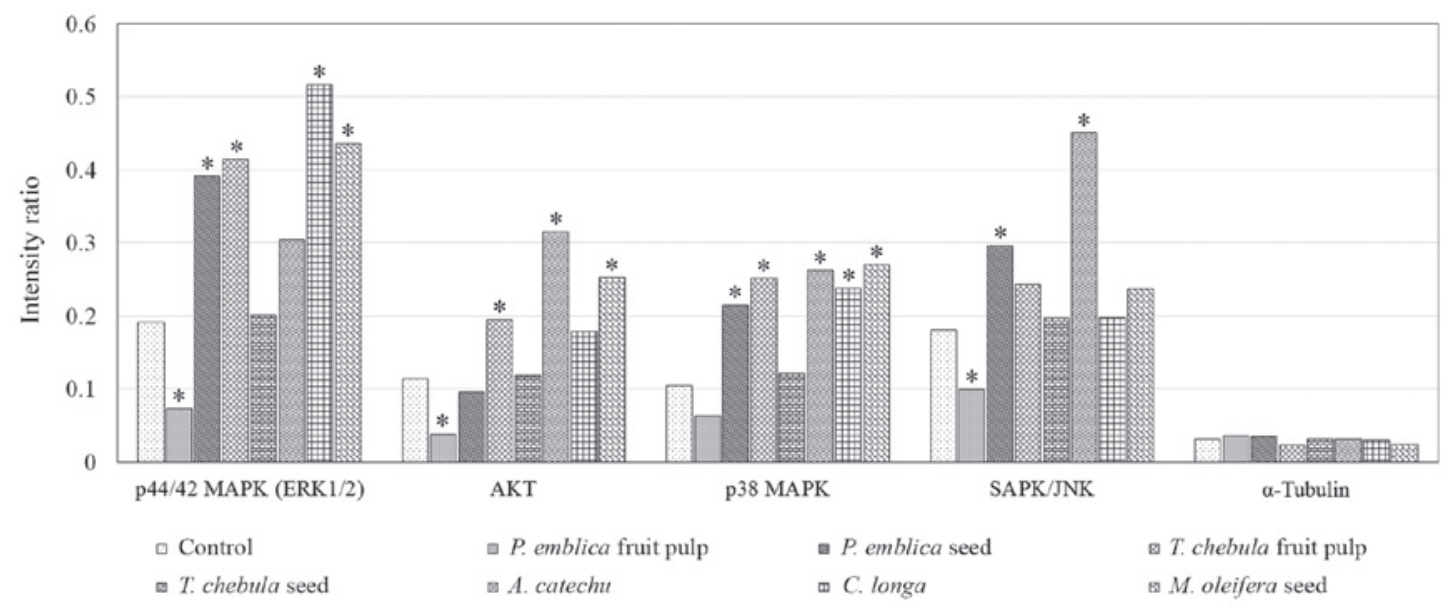

Figure 4. Detection of the phosphorylation of intracellular signal molecules in RMCCA1 cells treated with medicinal plant extracts. The phosphorylation levels of p44/42 MAPK (ERK1/2) at Thr202/Tyr204, Akt at Ser473, p38 MAPK at Thr180/Tyr182 and SAPK/JNK at Thr183/Tyr185 were significantly altered. The expression levels of $\alpha$-tubulin were used to normalize the signals between various samples. " $\mathrm{P}<0.05$ vs. control. MAPK, mitogen-activated protein kinase; Akt, protein kinase B; SAPK/JNK, stress-activated protein kinases/Jun amino-terminal kinases.

Increased activation of caspase-3 was demonstrated in RMCCA1 cells treated with the extracts of PEs, TCf, TCs, ACs, CL and MOs ( $\mathrm{P}=0.018,<0.001,0.018,0.032,0.029$ and 0.006, respectively); however, this was not observed in RMCCA1 cells treated with the extract of $\mathrm{PEf}(\mathrm{P}=0.183)$. No significant differences in caspase-7 expression levels were identified.

Other pro-apoptotic molecules, including cleaved-PARP, phosphorylated-Chk 2 and phosphorylated-p53, were demonstrated to be upregulated in RMCCA1 cells treated with certain plant extracts. The expression level of cleaved-PARP was significantly increased in RMCCA1 cells treated with the extracts of PEs, TCf, TCs, CL and MOs ( $\mathrm{P}=0.018,0.014$, 0.003, 0.039 and 0.005, respectively). Phosphorylated-Chk2 was significantly upregulated in RMCCA1 cells treated with the extracts of PEs, TCf, TCs, ACs and MOs ( $\mathrm{P}=0.048$, $0.005,0.012,0.029$ and 0.007 , respectively). The activation of phosphorylated-p53 was demonstrated in RMCCA1 cells treated with the extracts of PEs, TCf, TCs, ACs, CL and MOs $(\mathrm{P}=0.014,0.005,0.001,0.028,0.041$ and 0.002 , respectively; Fig. 3).

Activation of the Akt and MAPK pathways, including ERK1/2 and p38 MAPK, were demonstrated in RMCCA1 cells. The expression level of phosphorylated-ERK1/2 was demonstrated to be upregulated in RMCCA1 cells treated with the extract of PEs, TCf, CL and MOs ( $\mathrm{P}=0.010,0.003,<0.001$ and 0.001 , respectively) while RMCCA1 cells treated with the extracts of PEs, TCf, ACs, CL and MOs exhibited significantly increased expression levels of phosphorylated-p38 MAPK ( $\mathrm{P}=0.004,0.002,0.042,0.037$ and 0.002 , respectively). The expression level of phosphorylated-AKT was significantly upregulated in RMCCA1 cells treated with the extract of TCf, ACs and MOs ( $\mathrm{P}=0.001,0.034$ and <0.001, respectively). 
However, the expression level of phosphorylated-ERK1/2 was significantly downregulated in the RMCCA1 cells treated with PEf extract $(\mathrm{P}=0.005)$, while the expression level of phosphorylated-p38 MAPK appeared downregulated but was not significant $(\mathrm{P}=0.054)$. In addition, the expression of phosphorylated-SAPK/JNK in the RMCCA1 cells treated with PEf extract was significantly downregulated $(\mathrm{P}=0.004)$. The activation of SAPK/JNK was demonstrated in RMCCA1 cells treated with the extracts of PEs and ACs $(\mathrm{P}=0.043$ and 0.023, respectively; Fig. 4).

\section{Discussion}

The present study investigated the inhibition of CCA cell proliferation by various types of medicinal plants. Each plant extract was composed of mixture of bioactive compounds (19); however, the current study evaluated the activity of the entire extract rather than the activities of the individual compounds. Inhibition of CCA cell proliferation was associated with the upregulation of numerous intracellular signaling molecules and pro-apoptotic molecules. A total of 19 signaling molecules involved in the regulation of the stress response and apoptosis were simultaneously detected in the present study.

Caspase-3 protease exerts a pro-apoptotic function through the cleavage of multiple cellular targets, and is activated in apoptotic cells by extrinsic (death ligand) and intrinsic (mitochondrial) pathways (20). The present study demonstrated that caspase-3 is activated (via cleavage at Asp175) in CCA cells treated with the extracts of PEs, TCf, TCs, ACs, CL and MOs. The results revealed that these same extracts also inhibit PARP function via cleavage at Asp214. PARP is a DNA repair enzyme that is activated in response to DNA strand breaks (21). During apoptosis, cleavage of PARP-1 at Asp214 by caspase-3 is a useful marker of this type of cell death (21). The results of the current study suggested that the extracts of PEs, TCf, TCs, ACs, CL and MOs induced apoptosis in RMCCA1 cells.

The extracts of PEs, TCf, TCs, ACs and MOs were identified to increase Chk2 phosphorylation in CCA cells. Chk2 functions as a cell cycle checkpoint regulator and is a putative tumor suppressor protein; it is phosphorylated in response to DNA damage and prevents the cell from entering mitosis, thus arresting the cell cycle in the G1 phase (22). Previous studies have demonstrated that gallic acid (a hydrolysable tannin present in TCf) and quercetin induce cell cycle arrest in the G1 phase and induce apoptosis in certain cancer cells by inactivating the phosphorylation of $\mathrm{Cdc} 25 \mathrm{~A} / \mathrm{Cdc} 25 \mathrm{C}-\mathrm{Cdc} 2$ via Chk2 activation $(23,24)$. Therefore, the induction of $\mathrm{Chk} 2$ by the aforementioned plant extracts may have an important role in CCA apoptosis.

Accumulation of p53 has a crucial role in cell apoptosis. The phosphorylation of p53 underlies p53 accumulation and its subsequent prolonged protein half-life due to ubiquitination and degradation (25). The phosphorylation of $\mathrm{p} 53$ at Ser15 promotes the accumulation and functional activation of p53 in response to apoptotic stimuli or DNA damage (26). The current study demonstrated that p53 phosphorylation at Ser15 is increased in CCA cells treated with extracts of PEs, TCf, TCs, ACs, CL and MOs. Furthermore, it has previously been demonstrated that the phosphorylation of p53 at Ser15 may induce protein translocation to the mitochondria (intrinsic pathway), thereby inducing apoptosis by the mitochondrial pathway (27). Consequently, the present study found that the induction of p53 phosphorylation of at Ser15 in CCA cells treated with the extracts of PEs, TCf, TCs, ACs, CL and MOs, may also induce apoptosis by activating caspase- 3 which serve a critical role in the induction of apoptosis in mitochondrial pathway and inhibiting PARP function. Wu et al (28) identified that the silencing of p53 by specific small interfering RNA reduced the effect of CL on the induction of nasopharyngeal carcinoma cell apoptosis. Therefore, p53 function may be important to the ability of the specified plant extracts to induce CCA cell apoptosis.

ERK1/2 and p38 kinase are activated in CCA cells that have been treated with the extracts of PEs, TCf, ACs, CL and MOs. Our previous study demonstrated that ERK1/2 functions to promote survival in CCA cells, whereas p38 kinase activation is a primary signal for apoptosis (29). ERK1/2 signaling is required for the proliferation of CCA cells and the inhibition of ERK1/2 contributes to cell apoptosis (30). A previous study suggested that the opposing effects of ERK1/2 and p38 kinase in apoptosis are regulated by the phosphorylation and accumulation of p53 (31). In addition, p38 kinase may physically interact with and phosphorylate p53 at Ser15 in response to ultraviolet B radiation, and induce apoptosis in vitro and in vivo (32). The present study demonstrated that the simultaneous activation of p38 kinase and p53 could be observed in CCA cells treated with extracts of PEs, TCf, ACs, $\mathrm{CL}$ and MOs, which suggests that the interaction of p53 and p38 MAPK induces apoptosis in CCA cells.

The current study also demonstrated that JNK is activated in CCA cells that have been treated with extracts of PEs and ACs. Consistently, a previous study revealed that curcumin induced osteoclastoma cell apoptosis by activating the JNK signaling pathway (33). JNK belongs to the superfamily of MAPKs and has a critical role in death receptor-initiated (extrinsic) and mitochondrial (intrinsic) apoptotic pathways (34). The induction of apoptosis by PEs and ACs extracts may be regulated by the JNK pathway.

In the present study, PEf extract was demonstrated to inhibit CCA cell proliferation; however, apoptotic signals in the cells treated with PEf were not observed. We hypothesized that the anti-proliferative effect of PEf occurs via the inhibition of Akt activation. Akt is a protein kinase that promotes cell growth and survival (35), and our previous study demonstrated that Akt is involved in the regulation of CCA cell proliferation and invasion (36). The results of the present study are concordant with another previous study, which demonstrated that Phyllanthus extracts inhibit prostate cancer cell proliferation by inhibiting Akt activation (37).

The present study investigated the interaction of certain signaling molecules in CCA cells that have important roles in the regulation of apoptosis by specific plant extracts. Certain plants exhibited anti-proliferative effects in CCA cells by inducing pro-apoptotic signals; however, they also exhibited anti-apoptotic signals, including the phosphorylation of Bad at Ser136 and ERK1/2. Furthermore, the concentrations of the extracts required to inhibit CCA cells are relatively high, as the selected samples were from dietary medicinal plants. Further studies must be performed in in vivo to investigate the potential applications of these plant extracts in the clinical treatment of CCA. 


\section{Acknowledgements}

This study was funded by the Research Institute of Rangsit University (grant no., 37/2555). The manuscript underwent English language editing by editors at American Journal Experts.

\section{References}

1. Sripa B and Pairojkul C: Cholangiocarcinoma: Lessons from Thailand. Curr Opin Gastroenterol 24: 349-356, 2008.

2. Shanmugam MK, Kannaiyan R and Sethi G: Targeting cell signaling and apoptotic pathways by dietary agents: Role in the prevention and treatment of cancer. Nutr Cancer 63: 161-173, 2011.

3. Zhao T, Sun Q, Marques M and Witcher M: Anticancer properties of Phyllanthus emblica (Indian gooseberry). Oxid Med Cell Longev 2015: 950890, 2015.

4. Mahata S, Pandey A, Shukla S, Tyagi A, Husain SA, Das BC and Bharti AC: Anticancer activity of Phyllanthus emblica Linn. (Indian gooseberry): Inhibition of transcription factor AP-1 and HPV gene expression in cervical cancer cells. Nutr Cancer 65 (Suppl): S88-S97, 2013.

5. Zhu X, Wang J, Ou Y, Han W and Li H: Polyphenol extract of Phyllanthus emblica (PEEP) induces inhibition of cell proliferation and triggers apoptosis in cervical cancer cells. Eur J Med Res 18: 46, 2013.

6. Rahmani AH, Al Zohairy MA, Aly SM and Khan MA: Curcumin: A potential candidate in prevention of cancer via modulation of molecular pathways. BioMed Res Int 2014: $761608,2014$.

7. Kumar N, Gangappa D, Gupta G and Karnati R: Chebulagic acid from Terminalia chebula causes G1 arrest, inhibits $\mathrm{NF \kappa B}$ and induces apoptosis in retinoblastoma cells. BMC Complement Altern Med 14: 319, 2014.

8. Leelawat S and Leelawat K: Moringa oleifera extracts induce cholangiocarcinoma cell apoptosis by induction of reactive oxygen species production. Int J Pharmacog Phytochem Res 6 : 183-189, 2014.

9. Berkovich L, Earon G, Ron I, Rimmon A, Vexler A and Lev-Ari S: Moringa Oleifera aqueous leaf extract down-regulates nuclear factor-kappaB and increases cytotoxic effect of chemotherapy in pancreatic cancer cells. BMC Complement Altern Med 13: 212, 2013.

10. Nhiem NX, Yen PH, Ngan NT, Quang TH, Kiem PV, Minh CV, Tai BH, Cuong NX, Song SB and Kim YH: Inhibition of nuclear transcription factor- $\kappa \mathrm{B}$ and activation of peroxisome proliferator-activated receptors in HepG2 cells by cucurbitane-type triterpene glycosides from Momordica charantia. J Med Food 15: 369-377, 2012.

11. Li CJ, Tsang SF, Tsai CH, Tsai HY, Chyuan JH and Hsu HY: Momordica charantia extract induces apoptosis in human cancer cells through caspase- and mitochondria-dependent pathways. Evid-Based Complement Altern Med 2012: 261971, 2012.

12. Li M, Gao F, Zhou ZS, Zhang HM, Zhang R, Wu YF, Bai MH, Li JJ, Lin SR and Peng JY: Arecoline inhibits epithelial cell viability by upregulating the apoptosis pathway: Implication for oral submucous fibrosis. Oncol Rep 31: 2422-2428, 2014.

13. Devi JR and Thangam EB: Mechanisms of anticancer activity of sulforaphane from Brassica oleracea in HEp-2 human epithelial carcinoma cell line. Asian Pac J Cancer Prev 13: 2095-2100, 2012.

14. Abu MN, Salleh MAM, Radzman NHM, Ismail WIW, Yusoff RM and Hasan HF: Insulin sensitivity enhancement of the mixture of Tinospora Crispa and Gelam (Melaleuca Cajuputi) honey and its antiproliferative activity on hepatocellular carcinoma, HepG2: A preliminary study. J Med Res Devel 2: 48-54, 2013.

15. Yousafa Z, Wanga Y and Baydounc E: Phytochemistry and pharmacological studies on Solanum torvum Swartz. J Appl Pharm Sci 3: 152-160, 2013.

16. Mohammadi-Motlagh HR, Mostafaie A and Mansouri K: Anticancer and anti-inflammatory activities of shallot (Allium ascalonicum) extract. Arch Med Sci 7: 38-44, 2011.
17. Rattanasinganchan $\mathrm{P}$, Leelawat $\mathrm{K}$, Treepongkaruna SA Tocharoentanaphol C, Subwongcharoen S, Suthiphongchai T and Tohtong R: Establishment and characterization of a cholangiocarcinoma cell line (RMCCA-1) from a Thai patient. World J Gastroenterol 12: 6500-6506, 2006.

18. Nowsheen S and Yang ES: The intersection between DNA damage response and cell death pathways. Exp Oncol 34: 234-254, 2012.

19. Sasidharan S, Chen Y, Saravanan D, Sundram KM and Yoga Latha L. Extraction, isolation and characterization of bioactive compounds form plants' extracts. Afr J Tradit Complement Altern Med 8: 1-10, 2011.

20. Shalini S, Dorstyn L, Dawar S and Kumar S: Old, new and emerging functions of caspases. Cell Death Differ 22: 526-539, 2015.

21. Yu SW, Wang H, Dawson TM and Dawson VL: Poly(ADP-ribose) polymerase-1 and apoptosis inducing factor in neurotoxicity. Neurobiol Dis 14: 303-317, 2003.

22. Smith J, Tho LM, Xu N and Gillespie DA: The ATM-Chk2 and ATR-Chk1 pathways in DNA damage signaling and cancer. Adv Cancer Res 108: 73-112, 2010.

23. Suh DK, Lee EJ, Kim HC and Kim JH: Induction of G(1)/S phase arrest and apoptosis by quercetin in human osteosarcoma cells. Arch Pharm Res 33: 781-785, 2010.

24. Agarwal C, Tyagi A and Agarwal R: Gallic acid causes inactivating phosphorylation of cdc25A/cdc25C-cdc2 via ATM-Chk2 activation, leading to cell cycle arrest, and induces apoptosis in human prostate carcinoma DU145 cells. Mol Cancer Ther 5: 3294-3302, 2006.

25. Meek DW: Regulation of the $\mathrm{p} 53$ response and its relationship to cancer. Biochem J 469: 325-346, 2015.

26. Dmitrieva NI, Michea LF, Rocha GM and Burg MB: Cell cycle delay and apoptosis in response to osmotic stress. Comp Biochem Physiol A Mol Integr Physiol 130: 411-420, 2001.

27. Chi SW: Structural insights into the transcription-independent apoptotic pathway of p53. BMB Rep 47: 167-172, 2014.

28. Wu J, Tang Q, Zhao S, Zheng F, Wu Y, Tang G and Hahn SS: Extracellular signal-regulated kinase signaling-mediated induction and interaction of $\mathrm{FOXO} 3 \mathrm{a}$ and $\mathrm{p} 53$ contribute to the inhibition of nasopharyngeal carcinoma cell growth by curcumin. Int J Oncol 45: 95-103, 2014.

29. Sui X, Kong N, Ye L, Han W, Zhou J, Zhang Q, He C and Pan H: p38 and JNK MAPK pathways control the balance of apoptosis and autophagy in response to chemotherapeutic agents. Cancer Lett 344: 174-179, 2014.

30. Leelawat K, Keeratichamroen S, Leelawat S and Tohtong R: CD24 induces the invasion of cholangiocarcinoma cells by upregulating CXCR4 and increasing the phosphorylation of ERK1/2. Oncol Lett 6: 1439-1446, 2013.

31. Kim SJ, Ju JW, Oh CD, Yoon YM, Song WK, Kim JH, Yoo YJ, Bang OS, Kang SS and Chun JS: ERK-1/2 and p38 kinase oppositely regulate nitric oxide-induced apoptosis of chondrocytes in association with $\mathrm{p53}$, caspase-3, and differentiation status. J Biol Chem 277: 1332-1339, 2002.

32. Gong X, Liu A, Ming X, Deng P and Jiang Y: UV-induced interaction between $\mathrm{p} 38$ MAPK and p53 serves as a molecular switch in determining cell fate. FEBS Lett 584: 4711-4716, 2010.

33. Cao F, Liu T, Xu Y, Xu D and Feng S: Curcumin inhibits cell proliferation and promotes apoptosis in human osteoclastoma cell through MMP-9, NF- $\mathrm{KB}$ and JNK signaling pathways. Int J Clin Exp Pathol 8: 6037-6045, 2015.

34. Nishina H, Wada T and Katada T: Physiological roles of SAPK/JNK signaling pathway. J Biochem 136: 123-126, 2004.

35. Davis WJ, Lehmann PZ and Li W: Nuclear PI3K signaling in cell growth and tumorigenesis. Front Cell Dev Biol 3: 24, 2015.

36. Leelawat K, Udomchaiprasertkul W, Narong S and Leelawat S: Induction of MKP-1 prevents the cytotoxic effects of PI3K inhibition in hilar cholangiocarcinoma cells. J Cancer Res Clin Oncol 136: 1537-1544, 2010.

37. Tang YQ, Jaganath I, Manikam R and Sekaran SD: Phyllanthus suppresses prostate cancer cell, PC-3, proliferation and induces apoptosis through multiple signaling pathways (MAPKs, PI3K/Akt, NFאB, and hypoxia). Evid-Based Complement Altern Med 2013: 1-13, 2013. 